\title{
Role of dental practitioner in smoking cessation interventional programs
}

\author{
Rajeh Mohammed Al-Sharif ${ }^{1 *}$, Mohamed Ibrahim Homadi², Huda Abdullah Alsakran ${ }^{3}$, \\ Mohammed Ahmed Alqarni ${ }^{4}$, Amr Hamdi Almaliki², Elaf Ahmed Alkuwaiti ${ }^{5}$, \\ Dina Abdulwahab Bawahab ${ }^{6}$, Rashid Abdullah Alshehri' ${ }^{2}$, Azza Anas Abudawood ${ }^{7}$, \\ Abdulaziz Talal Albishri ${ }^{8}$, Alyyan Mohammed Alqahtani ${ }^{2}$
}

\author{
${ }^{1}$ North Jeddah Specialist Dental Center, King Abdullah Medical Complex, Jeddah, Saudi Arabia \\ ${ }^{2}$ College of Dentistry, King Khalid University, Abha, Saudi Arabia \\ ${ }^{3}$ Dental Department, Prince Sultan Military Medical City, Riyadh, Saudi Arabia \\ ${ }^{4}$ General Dentist, Allure Medical Center, Riyadh, Saudi Arabia \\ ${ }^{5}$ College of Dentistry, Imam Abdulrahman Bin Faisal University, Dammam, Saudi Arabia \\ ${ }^{6}$ General Dentist, Round Dental Clinics, Jeddah, Saudi Arabia \\ ${ }^{7}$ General Dentist, King Abdullah Medical Complex, Jeddah, Saudi Arabia \\ ${ }^{8}$ College of Dentistry, Vision Colleges, Jeddah, Saudi Arabia
}

Received: 20 December 2021

Accepted: 05 January 2022

*Correspondence:

Dr. Rajeh Mohammed Al-Sharif,

E-mail: dr.rmsh@hotmail.com

Copyright: () the author(s), publisher and licensee Medip Academy. This is an open-access article distributed under the terms of the Creative Commons Attribution Non-Commercial License, which permits unrestricted non-commercial use, distribution, and reproduction in any medium, provided the original work is properly cited.

\begin{abstract}
Oral cancer, periodontitis, reduced response to periodontal therapy, implant failure, tooth loss, dental caries, delayed wound healing, chronic candidiasis, staining, halitosis and premalignant tissue alteration are all caused or linked to smoking and smokeless tobacco use. Importantly, smoking cessation therapies (both brief and extensive) have been proved to be a very cost-effective method of prolonging life and lowering disease. Health care providers, notably dentists and dental hygienists, are responsible for providing smoking cessation care to individuals with oral difficulties. The purpose of this research was to review the available information about the effectiveness of dental practitioners in smoking cessation interventional programs. According to the existing literature, dental practitioners can play an essential role in the control and spread of tobacco-related oral disorders by including smoking cessation interventional programs. The negative health impacts of tobacco use as well as the benefits of quitting tobacco use and tobacco cessation counselling, should all be part of the dentist office's arsenal. Dental professionals are in a unique position to assist tobacco users seeking dental care by assisting them in quitting smoking.
\end{abstract}

Keywords: Dental practitioner, Smoking cessation, Oral cancer, Tobacco use, Interventional programs

\section{INTRODUCTION}

In developed countries, smoking is one of the most serious public health issue. Tobacco kills around 6 million people each year and costs the global economy more than half a trillion dollars. ${ }^{1}$ Oral cancer, gum disease and other oral health issues are caused by cigarettes, smokeless tobacco and other tobacco products. People who smoke cigarettes have a higher rate of untreated dental decay. Untreated tooth decay affects more than $40 \%$ of persons aged 20 to 64 who use cigarettes. Health care workers in several settings play an important role in assisting people who want to quit smoking. ${ }^{2}$ Oral cancer kills about 140,000 people every year around the world. Oral cancer affects more than 300,000 people each year. In the United Kingdom, the rate of oral cancer has risen by 68 percent in the last 20 years. Tobacco smoking or other forms of tobacco use 
cause most oral malignancies. ${ }^{3}$ These oral problems are caused or related with smoking and smokeless tobacco use: delayed wound healing, chronic candidiasis, staining, and halitosis; band premalignant tissue change; implant failure; tooth loss; dental caries; delayed wound healing, chronic candidiasis, staining and halitosis. ${ }^{4}$

Importantly, smoking cessation therapies (both brief and extensive) have been proved to be a very cost-effective method of prolonging life and lowering disease burden. All smoking cessation therapies (short and more intense, as well as those including medication) have low costs per quality adjusted life year (QALY). The QALY is a measure of illness burden that considers both the quality and quantity of life lived and it is used to evaluate the cost-effectiveness of health-care treatments. ${ }^{5}$ Smoking is now widely recognized as a risk factor for a variety of oral illnesses. Health care providers, notably dentists and dental hygienists are responsible for providing smoking cessation care to individuals with oral difficulties. By including smoking cessation interventional programs, dental practitioners can play a critical role in controlling and spreading oral disease associated with tobacco use.

The purpose of this research was to review the available information about the effectiveness of role of dental practitioners in smoking cessation interventional programs.

\section{Methodology}

This study was based on a comprehensive literature search conducted on 20 December 2021 in the Medline and Cochrane databases, utilizing the medical topic headings $(\mathrm{MeSH})$ and a combination of all available related terms, according to the database. To prevent missing any possible research, a manual search for publications was conducted through Google Scholar, using the reference lists of the previously listed papers as a starting point. We looked for valuable information in papers that discussed smoking cessation techniques in dentistry settings. There were no restrictions on date, language, participant age or type of publication.

\section{DISCUSSION}

Although the negative effects of tobacco on periodontal tissues have been well documented, little was known about the potential benefits of quitting smoking on periodontal health. ${ }^{6}$ Since dentists and dental hygienists were excellent at treating tobacco use and dependence, identifying, documenting and treating every tobacco user they encountered it should become a standard practice in every dental office and clinic. According to Scott tobacco intervention must be considered a necessary component of high-quality dental treatment. ${ }^{7}$

The consequences of tobacco usage on public oral health were equally concerning. Tobacco products including cigarettes, cigars, pipes and smokeless tobacco have been proven to cause oral and pharyngeal cancer and were responsible for more than $75 \%$ of cancer-related deaths in the United States. ${ }^{8}$

The data was strong enough to consider smoking as a cause of adult periodontitis, with one-half of all instances in the United States being linked to cigarette smoking. Tobacco smoking significantly lowered the prognosis of periodontal therapy and dental implants, slowed oral wound healing and raised the likelihood of a variety of oral soft tissue alterations in the patient. ${ }^{9}$ The American dental association's survey of current issues in dentistry, published in 1997, found that cessation of tobacco use efforts more than four out of ten dentists do not consistently enquire about tobacco use (a figure that has remained almost stable since 1994) and six out of ten dentists did not routinely counsel tobacco users to quit. ${ }^{10}$ Unfortunately, just $24 \%$ of smokers who had seen a dentist in the previous year said their dentist had urged them to quit, and only $18 \%$ of smokers who used smokeless tobacco said their dentist had ever advised them to quit. ${ }^{11}$

Patients who visited the dentist for regular checks were especially responsive to health messaging and the oral effects of tobacco use gave visible evidence and a powerful motivation for smokers to stop. The recently released US public health service (PHS) recommendations for treating tobacco addiction and dependence equip dentists and other primary care clinicians with evidence-based, practical techniques to adopted into their practices. ${ }^{12}$ Despite the fact that tobacco usage had decreased in some parts of the world, it remained a persistent and in other circumstances, developing problem that will continue to be a major challenge for dentists in the next decades. Dental professionals have a unique opportunity and professional commitment to help reduce the economic and social costs of tobacco use's impact on dental and general health. Dental practitioners can utilize non-invasive, evidencebased treatments to help patients avoid starting tobacco use, encourage and assist patients in quitting tobacco use and address tobacco-induced damage to periodontal supporting tissues. ${ }^{13}$ The link between tobacco use and oral health had long been acknowledged by organized dentistry as has the need for oral health practitioners to treat tobacco use. The American dental association encourages dentists to help patients quit smoking. ${ }^{14}$

In a study conducted by Gordan in which dentists, dental hygienists and dental assistants in the intervention condition received a 3-hour in-service workshop on integrating and delivering a brief, tailored tobacco intervention to their patients including proper use of nicotine replacement therapy. ${ }^{15}$ The results showed that participants in the intervention group reported significantly higher abstinence rates at the 7.5-month follow up, for both point prevalence and lifetime prevalence. According to a study conducted in India, most dentists $(98.7 \%)$ thought that providing smoking 
cessation counselling was their job. ${ }^{16}$ Lack of time was cited by 54.3 percent of dentists as a key impediment to smoking cessation counselling implementation. Almost 37 percent said they didn't know enough about the topic. If patients were too coached, $35.8 \%$ were concerned that they might leave their clinic. However, in addition to the dentists' positive attitudes, some impediments to providing smoking cessation counselling to patients were discovered. The most common hurdles found were a lack of time, a lack of understanding and a fear that patients would leave the clinic. For those attempting to adopt them, a lack of time was a key hindrance.

According to the findings of the Eva et al study in Sweden, at the 12-month follow up, abstinence rates were $8 \%$ higher in both programs: high intensity treatment and low intensity treatment in a dental setting for smoking cessation. ${ }^{17}$ At the 5 to 8 year follow up, 27 percent of the individuals had quit smoking.

Available evidence suggested that oral health professionals conducting tobacco cessation treatments in the dental office or community context with an oral examination component may boost tobacco abstinence rates among both cigarette smokers and smokeless tobacco users. Because of the differences between the studies, it's difficult to make definitive recommendations about which intervention components should be used in clinical practice; however, behavioural counselling (usually brief) in conjunction with an oral examination was a consistent intervention component that was also provided in some control groups. ${ }^{18}$ Tobacco smokers who received treatment in the dental environment were more likely to quit. However, the majority of the evidence came from patients who used smokeless tobacco. In medical settings, pharmacotherapy (such as nicotine substitutes, bupropion and varenicline) was indicated for TUC (tobacco use cessation), but little research had been done in dental settings, despite encouraging evidence to date. ${ }^{19}$

Tobacco use had long been recognized as a substantial risk factor for periodontal disease development and progression. Increased pocket depths, loss of periodontal attachment, alveolar bone loss and a higher rate of tooth loss have all been linked to its use. Nicotine, a major component and the most pharmacologically active substance in tobacco, was believed to be a substantial contributor to periodontal disease aggravation. According to the available literature, nicotine affected gingival blood flow, cytokine generation, neutrophil and other immune cell activity as well as connective tissue turnover, which could be the processes behind tobacco's overall impacted on periodontal tissues. When tobacco cessation was included as part of periodontal care, dental professionals were more likely to become involved in tobacco cessation counselling. This will have a significant positive impact on the oral and general health of our patients. ${ }^{20}$
The relationship between smoking and periodontal diseases had been extensively studied over the last 15 years, with strong epidemiologic evidence of a positive association between smoking and clinical and radiographic signs of periodontitis as well as an increased risk of periodontitis in smokers, from both cross-sectional and longitudinal studies. Smoking was a strong predictor of future bone loss in a 10 year longitudinal radiography investigation of alveolar bone loss in those participants who had at least 20 teeth at the start of the trial. ${ }^{21,22}$ According to a study performed in Japan, there was a need for health practitioners to promote smoking cessation efforts more aggressively. ${ }^{23}$ The data also implied that, although having a role in smoking care, dentists and dental hygienists required training in providing smoking cessation therapy to hospital patients. To overcome the potential barriers, it was vital to provide adequate training to employees and to create an environment that supported smoking cessation efforts.

Smoking had long been recognized as a risk factor for a variety of oral disorders including oral cancer and periodontal disease. Oral health specialists were in a unique position to help smokers quit by offering appropriate advice on the numerous facets of tobaccorelated diseases. Dentists and dental hygienists have frequent contact with smokers and have a lot of potential to help them quit, however, this potential was frequently misused. ${ }^{24}$ When compared to western developed countries, it had also been stated that Japanese health professionals were less aware of their function as role models for dental patients. ${ }^{25,26}$

Oral health experts can help their patients quit smoking by supporting them and referring them to resources. Dental personnel should also be trained and provided the resources they need to offer the advice. A quarter of tobacco smokers in the United States had been counselled to stop by their dentist by the mid-1990s..$^{27,28}$ A Cochrane review of smoking cessation training for health workers found that those who were taught were better at administering smoking cessation programs. There were no statistically significant connections between the obstacles and demographic factors such as years in practice in this investigation. ${ }^{29}$

In Europe, 29 percent of people call themselves smokers, and tobacco use was directly linked to 650,000 fatalities per year or around 14 percent of all deaths. From a dentistry standpoint, smoking was linked to a number of chronic and potentially life-threatening conditions that only affected the mouth cavity. It was possible to ensure that smokers were assisted at every opportunity by familiarizing the dental team with gold-standard cessation counselling. Dentists should try to give smokers cessation counselling based on their willingness to stop their smoking habits. $^{30}$ Only employ pharmacological assistance if the patient had already failed to quit smoking, according to Carlos et al. ${ }^{31}$ The "5 As" strategy should be used in conjunction with NRT (nicotine 
replacement therapy), rather than pharmacological treatment, which should be avoided at first depending on the patient's level of addiction and should always be used in conjunction with behavioural therapy, motivational counselling and alternative therapies if the patient chooses to do so as a complementary exercise. Finally, dental experts were in a unique position to detect the consequences of smoking on oral health early on, as well as provide smokers with recommendations and information on how to avoid or quit smoking. They should provide quick, concise and patient-specific advice and assistance.

\section{CONCLUSION}

The dangers of smoking on one's overall and dental health are well-known. According to studies, dentists educated in smoking cessation counselling were able to contribute to community-based smoking cessation programs with success rates comparable to those found in general medical practices. The negative health impacts of tobacco use, as well as the benefits of quitting tobacco use, and tobacco cessation counselling, should all be part of the dentist office's arsenal. Dental professionals are in a unique position to assist tobacco users who seek dental care in quitting smoking or abstaining from other tobacco products. However, because most clinical and epidemiological studies were conducted in the past, current research on the role of dental practitioners in smoking cessation interventional programs should be conducted to demonstrate the value and define future recommendations as needed.

\section{Funding: No funding sources}

Conflict of interest: None declared

Ethical approval: Not required

\section{REFERENCES}

1. WHO report on the Global Tobacco Epidemic, 2021. Available at: https://apps.who.int/iris/ bitstream/handle/10665/343287/9789240032095eng.pdf. Accessed on 3 July 2021.

2. Centres for Disease Control and Prevention. Oral Health Surveillance Report: Trends in Dental Caries and Sealants, Tooth Retention, and Edentulism, United States, 1999-2004 to 2011-2016. Atlanta, GA: Centres for Disease Control and Prevention, US Dept. of Health and Human Services; 2019. Available at: https://www.cdc.gov/oralhealth/ publications/OHSR-2019-index.html. Accessed on 3 July 2021.

3. Chaturvedi P, Singh A, Chien C, Warnakulasuriya S. Tobacco related oral cancer. Brit Med J. 2019;365:2142.

4. Brothwell DJ, Armstrong KA. Smoking cessation services provided by dental professionals in a rural Ontario health unit. J Canadian Dent Assoc. 2004;70(2):94-8.
5. National Institute for Clinical Excellence (NICE). Measuring effectiveness and cost-effectiveness: the QALY. April 2010. Available at: https://www.nice. org.uk/process/pmg6/chapter/assessing-costeffectiveness. Accessed on 3 July 2021.

6. Fiorini T, Musskopf ML, Oppermann RV, Susin C. Is there a positive effect of smoking cessation on periodontal health? A systematic review. J Periodontol. 2014;85(1):83-91.

7. Tomar SL. Dentistry's role in tobacco control. J Am Dent Assoc. 2001;132:30-5.

8. National Center for Chronic Disease Prevention and Health Promotion (US) Office on Smoking and Health. The Health Consequences of Smoking-50 Years of Progress: A Report of the Surgeon General. 1989.

9. Position paper: tobacco use and the periodontal patient. Research, science and therapy committee of the American academy of periodontology. Periodontol. 1999;70(9):1419-27.

10. American Dental Association. 1997 survey of current issues in dentistry: tobacco use cessation efforts among dentists. Chicago: ADA Survey Center; 1998.

11. Tomar SL, Husten CG, Manley MW. Do dentists and physicians advise tobacco users to quit. JADA. 1996;127(2):259-65.

12. Fiore M, Bailey WC, Cohen SJ. Treating tobacco use and dependence: clinical practice guideline. Rockville Md: U. S. Department of Health and Human Services, Public Health Service; 2000.

13. Chaffee BW, Couch ET, Ryder MI. The tobaccousing periodontal patient: role of the dental practitioner in tobacco cessation and periodontal disease management. Periodontol 2000. 2016;71(1):52-64.

14. American Dental Association. Summary of policy and recommendations regarding tobacco 1964present. 2009.

15. Gordon JS, Andrews JA, Albert DA, Crews KM, Payne TJ, Severson HH. Tobacco cessation via public dental clinics: results of a randomized trial. Am J Public Health. 2010;100(7):1307-12.

16. Bhat N, Jyothirmai-Reddy J, Gohil M, Khatri M, Ladha M, Sharma M. Attitudes, practices and perceived barriers in smoking cessation among dentists of Udaipur city, Rajasthan, India. Addict Health. 2014;6(1-2):73-80.

17. Nohlert E, Öhrvik J, Tegelberg A, Tillgren P, Helgason AR. Long-term follow-up of a high- and a low-intensity smoking cessation intervention in a dental setting: a randomized trial. BMC Public Health. 2013;13:592.

18. Carr AB, Ebbert J. Interventions for tobacco cessation in the dental setting. Cochrane Database Syst Rev. 2012;2012(6):005084.

19. Vivian IG, Ainamo BA, Ainamo A, Carr AB, Fundak A, Koerber A, et al. Improving the effectiveness of tobacco use cessation (TUC). Int Dent J. 2010;60(1):50-9. 
20. Malhotra R, Kapoor A, Grover V, Kaushal S. Nicotine and periodontal tissues. J Indian Soc Periodontol. 2010;14(1):72-9.

21. Darby ML, Walsh MM. Tobacco cessation. Dental hygiene theory and practice. 2nd ed. Philadelphia: W. B. Saunders; 2003.

22. Bolin A, Lavstedt S, Frithiof L, Henrikson CO. Proximal alveolar bone loss in a longitudinal radiographic investigation: IV: smoking and some other factors influencing the progress in individuals with at least 20 remaining teeth. Acta Odontol Scand. 1986;44(5):263-9.

23. Saito A, Nishina M, Murai K, Mizuno A, Ueshima F, Makiishi T, et al. Health professional's perceptions of and potential barriers to smoking cessation care: a survey study at a dental school hospital in Japan. BMC Res Notes. 2010;3:329.

24. Gonseth S, Abarca M, Madrid C, Cornuz J. A pilot study combining individual-based smoking cessation counselling, pharmacotherapy, and dental hygiene intervention. BMC Pub Health. 2010;10:348.

25. Ohida T, Sakurai H, Mochizuki Y, Kamal AM, Takemura S, Minowa M, et al. Smoking prevalence and attitudes toward smoking among Japanese physicians. JAMA. 2001;285(20):2643-8.

26. Naito T, Miyaki K, Naito M, Yoneda M, Suzuki N, Hirofuji $\mathrm{T}$, et al. Parental smoking and smoking status of Japanese dental hygiene students: a pilot survey at a dental hygiene school in Japan. Int $\mathbf{J}$ Environ Res Public Health. 2009;6(1):321-8.

27. Binnie VI, McHugh S, Jenkins W, Borland W, Macpherson LM. A randomised controlled trial of a smoking cessation intervention delivered by dental hygienists: a feasibility study. BMC Oral Health. 2007;7:5-10.

28. Martin LM, Bouquot JE, Wingo PA, Heath CW. Cancer prevention in the dental practice: oral cancer screening and tobacco cessation advice. J Public Health Dent. 1996;56(6):336-40.

29. Lancaster T, Stead L, Silagy C, Sowden A. Effectiveness of interventions to help people stop smoking: findings from the Cochrane Library. BMJ. 2000;321(7257):355-8.

30. Keat RM, Fricain J, Catros S, et al. The dentist's role in smoking cessation management: a literature review and recommendations: part 1 . Dent Update. 2018;45(3).

31. Omaña-Cepeda C, Jané-Salas E, Estrugo-Devesa A, Chimenos-Küstner E. Effectiveness of dentist's intervention in smoking cessation: a review. J Clin Experiment Dentist. 2016;8(1):78-83.

Cite this article as: Al-Sharif RM, Homadi MI, Alsakran HA, Alqarni MA, Almaliki AH, Alkuwaiti EA, et al. Role of dental practitioner in smoking cessation interventional programs. Int J Community Med Public Health 2022;9:1103-7. 